\title{
Vulnerability in Regionally Ischemic Human Heart. Effect of the Extracellular Potassium Concentration
}

\author{
Andrés Mena Tobar ${ }^{1,2}$, Jose M Ferrero ${ }^{3}$, Jose F Rodriguez Matas ${ }^{4, *}$. \\ 1 I3A, University of Zaragoza, Spain \\ 2 CIBER-BBN, Zaragoza, Spain \\ 3 Polytechnic University of Valencia, Spain \\ 4 Chemistry, Materials, and Chemical Engineering Department "Giulio Natta", Politecnico di \\ Milano, Italy
}

* Correspondence: josefelix.rodriguezmatas@polimi.it, Chemestry, materials and chemical engineering department "Giulio Natta", Politecnico di Milano, Milano, Italy.

\section{Introduction}

When dealing with the pathological heart, ventricular tachycardia and fibrillation are known to be two types of cardiac arrhythmias that usually take place during acute ischemia, and frequently lead to sudden death [1]. Even though these arrhythmias arise from different conditions, ischemia is the most important perpetrator among them. During ischemia, the delivery of nutrients to the myocardium diminishes, causing metabolic changes, which result in a progressive deterioration of the electric activity in the injured region [2]. These metabolic changes are mainly hypoxia, increased concentrations of the extracellular potassium $\left[\mathrm{K}^{+}\right]_{\text {o }}$ (hyperkalemia), decrease of intracellular ATP (hypoxia), and acidosis [3]. From an electrophysiological point of view, these metabolic changes imply alterations in the action potential (AP), excitability, conduction velocity (CV), and effective refractive period (ERP) among others, creating a substrate for arrhythmias and fibrillation $[3,4]$. In addition, the impact of ischemia in the myocardium is characterized by a high degree of heterogeneity both intramurally and transmurally. In the tissue affected by acute ischemia, two zones can be distinguis- hed: i) the central ischemic zone (CIZ) corresponding to the core of the tissue suffering from the lack of blood, and ii) a border zone (BZ) which comprises changes in electrophysiological properties between the healthy and ischemic regions [5]. Pro-arrhythmic mechanisms of acute ischemia have been extensively investigated, although often in animal models rather than in human ventricles. Seminal studies by Janse et al. $[4,6]$ in pig and dog hearts highlight the complexity of the pro- arrhythmic and spatio-temporally dynamic substrate in acute ischemia. Heterogeneity in excitability and repolarization properties across the border zone leads to the establishment of reentry around the ischemic region following ectopic excitation [4,7]. The same studies also showed intramural reentry in certain cases (highlighting the potential variability in the mechanisms). However, the mechanisms that determine reentry formation and intramural patterns in acute ischemia in the three-dimensional human heart remain unclear, due to low resolution of intramural recordings. In this work, we have studied the different propagation patterns and determined the vulnerable window in a human heart during acute ischemia.

\section{Materials and Methods}

The electrical activity of the heart was modelled by means of the well established monodomain equation [8]

$$
\begin{gathered}
\boldsymbol{\nabla} \cdot(\mathbf{D} \nabla V)=C_{m} \frac{\partial V}{\partial t}+J_{i o n}(V, \mathbf{u})+J_{s t m} \\
\frac{\partial \mathbf{u}}{d t}=\mathbf{f}(\mathbf{u}, V, t)
\end{gathered}
$$

where $V$ is the transmembrane potential, $\mathbf{D}$ is the second order anisotropic conductivity tensor, $C_{m}$ the membrane capacitance, $J_{\text {ion }}$ the ionic current, $J_{\text {stm }}$ the stimulus current, and u is a set of state variables associated with the ionic model. The set of equations (1) is subject to the zero flux boundary conditions.

$$
\mathbf{n} \cdot(\mathbf{D} \nabla \mathrm{V})=0
$$


In this work, the electric activity of the cell was modelled using the tenTusscher model (TP06) [9]. In simulating ischemia, the value of the parameters affected by ischemia were set to the experimental values corresponding to approximately ten minutes of ischemia. Hypoxia was considered by incorporating the ATP-sensitive $\mathrm{K}^{+}$current $\left(\mathrm{I}_{\mathrm{K}(\mathrm{ATP})}\right)$ to the TP06 model. The formulation proposed by Ferrero et al [10] was adopted and modified to account for the different cell types (epicardial, endocardial and midmyocardial) [11].

The ischemic region was located in the anterior side of the left ventricle mimicking the occlusion of the circumflex artery. The ischemic region was composed of realistically dimensioned transitional border zones (BZ), a normal zone (NZ), and the central zone (CZ) of ischemia. In the $\mathrm{CZ},\left[\mathrm{K}^{+}\right]_{\text {o }}$ was set to $9.9 \mathrm{mM}[5,10]$, the inward $\mathrm{Na}^{+}$and L-type $\mathrm{Ca}^{2+}$ currents were scaled by a factor of 0.85 to imitate acidosis $[13,14]$, and $[A T P]_{i}$ and $[A D P]_{i}$ concentrations were set to $5 \mathrm{mM}$ and $99 \mathrm{mM}$ respectively [6]. In addition, a $1.0 \mathrm{~mm}$ wash-out zone in the endocardium was incorporated as proposed in Wilensky et al. [7]. Realistic cell heterogeneity and fiber anisotropy were considered in the model [11]. Fig. 1a shows the finite element model of the human heart and a detail of the considered ischemic region.

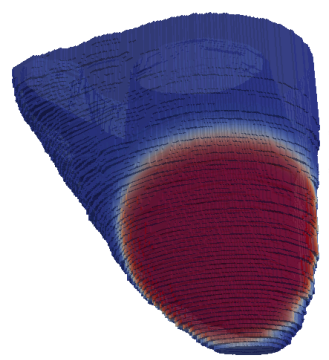

(a)

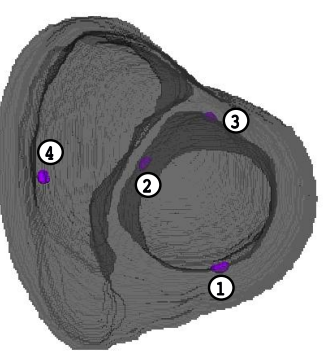

(b)

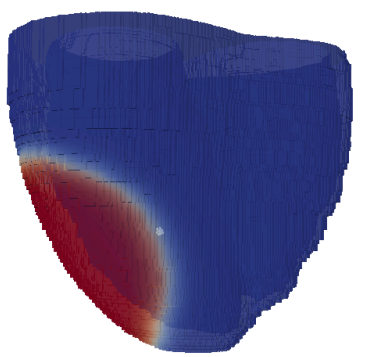

(c)

Figure 1. The computational model a) Detail of the ischemic zone, b) location of the normal stimulation sites, c) location of the ectopic stimulation.

The model was preconditioned for 45 seconds, stimulating at a frequency of $1.25 \mathrm{~Hz}$ at normal stimulation points as pointed in [15](see Fig. 1b). Following the preconditioning, an extra-stimulus located in the subendocardial border zone was delibered [6] (see Fig. 1c), with different coupling intervals $(\mathrm{CI})$. The reentrant patterns were studied for five seconds after the ectopic stimulus. Reentry patterns were investigated for different values of extracellular potassium concentration namely: $\left[\mathrm{K}^{+}\right]_{\mathrm{o}}$ $=(7.0,8.0,9.0)$. The vulnerability window defined as the range of CI's for which a re-entrant activity develops, was determined for each condition of $\left[\mathrm{K}^{+}\right]_{\mathrm{o}}$. Computations were performed on a GPU Tesla M2090 (6GRAM DDR5) with the software TOR [16] using the operator splitting and a semi-implicit scheme with a fixed time step of $0.02 \mathrm{~ms}$

\section{Results}

Results show spatial heterogeneities in the propagated action potential, as reported experimentally, throughout the regional ischemic tissue, such as resting membrane potential $(-85.2 \mathrm{mV}$ in NZ, and $72.5 \mathrm{mV}$ in the CZ, with potentials varying between these values in the BZ). During a basic beat, activity spreads from two directions into the ischemic area, whereas after the premature beat, activity spreads from the right side of the border into the ischemic area as shown in Fig. 2.

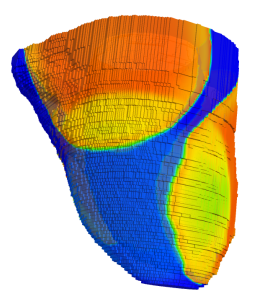

(a)

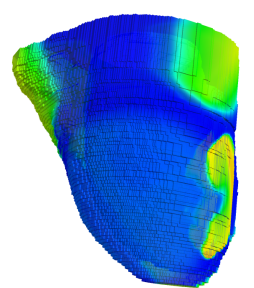

(b)

Figure 2: Depolarization pattern through the ischemic area during basic activation (left panel), and right after the ectopic beat (right panel). 
The vulnerability window for each value of $\left[\mathrm{K}^{+}\right]_{\mathrm{o}}$ is shown in Fig. 3. For CIs within the range shown in Fig. 3 reentry occurred for at least five seconds for $\left[\mathrm{K}^{+}\right]_{0}=7.0 \mathrm{nM}$; no sustained rentry (reentry pattern self extinguished within five seconds after delivering the ectopic stimulation) was obtained for $\left[\mathrm{K}^{+}\right]_{0}=8.0 \mathrm{nM}$; whereas for $\left[\mathrm{K}^{+}\right]_{0}=9.0 \mathrm{nM}$ no reentrant activity was found. In addition, within this window, the reentrant pattern was not altered by the presence of normal sinus rhythm stimulation.

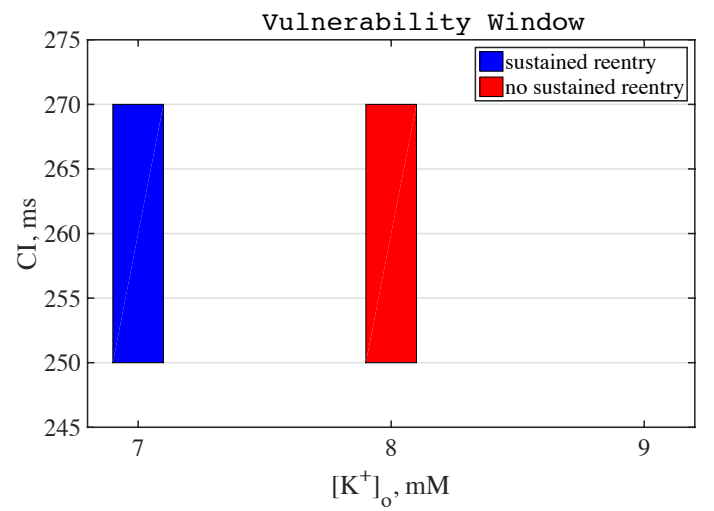

Figure 3. Vulnerability window for different values of $\left[\mathrm{K}^{+}\right]_{\mathrm{o}}$.

For CIs bellow the lower bound of the vulnerability window double blocked occurred without generating re-entrant activity. On the contrary, for CI's above the upper limit of the vulnerable window, conduction through the ischemic zone occurred. Within the vulnerable window, re-entrant activity originated in the endocardium as a single rotor, that propagated transmurally toward the epicardium as shown in Fig. 4 for $\mathrm{CI}=270 \mathrm{~ms}$ and $\left[\mathrm{K}^{+}\right]_{0}=7.0 \mathrm{nM}$.

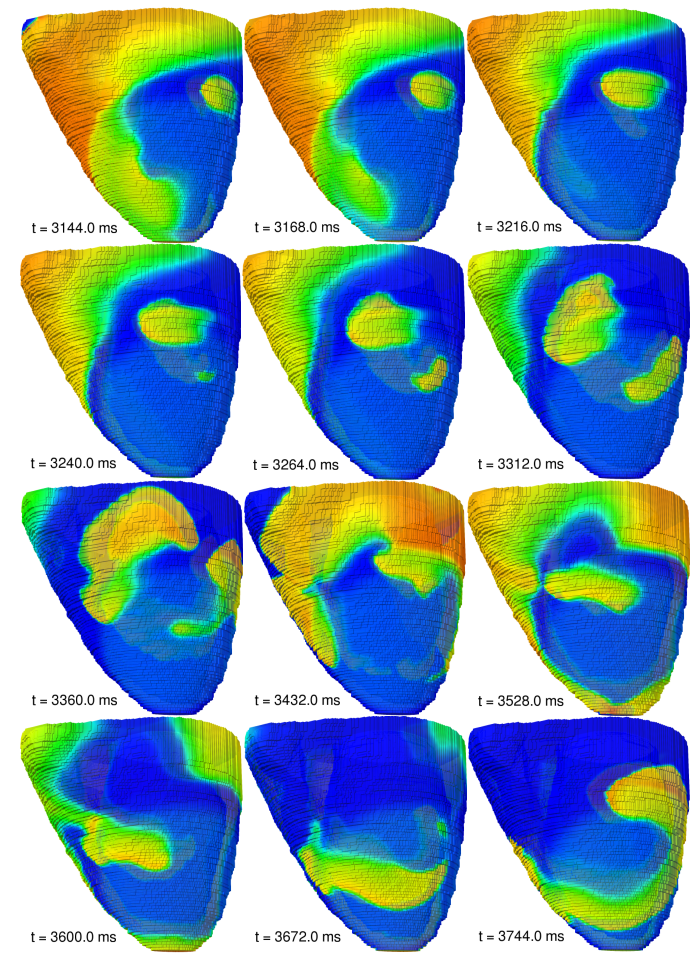

Figure 4. Snapshots of the reentrant activity for a $\mathrm{CI}=270 \mathrm{~ms}$ and $\left[\mathrm{K}^{+}\right]_{\mathrm{o}}=7.0 \mathrm{nM}$. The pattern of reentry changed from a single rotor to a figure of eight, and again to a single rotor.

Even though the reentrant activity always started in the endocardium in the form of a rotor, the pattern of the reentrant wavefront changed depending on the CI. For CIs between $250 \mathrm{~ms}$ and $270 \mathrm{~ms}$, reentrant patterns consisting of a single rotor and figure of eight were observed as shown in the sequence of pictures in Fig. 4 for $\mathrm{CI}=270 \mathrm{~ms}$. In the instant shown, the pattern changed from a single 
rotor, to a figure of eight, to then return to a single rotor. The reentry was always observed within the ischemic region. As the CI increased, the reentrant pattern was found to evolve towards a more stable configuration consisting in a single rotor meandering within the ischemic region. In all cases it was found that the reentrant front was lead from the endocardial surface where the pattern was clearly identified for all CIs. The reentry patterns were not affected when the basic stimulation was maintained after delivering the extra-stimulus.

\section{Discussion and Conclusions}

The main results of the simulations can be summarized as follows: i) As a consequence of the applied extra-stimulus that originates an ectopic beat, reentrant activity generated for CIs which range depends on the value of the extracellular potassium concentration $\left[\mathrm{K}^{+}\right]_{0}$; ii) The reentrant activity generated due to the extra-stimulus initiated as a consequence of the interaction between wavefronts emerging from the wash-out zone into the ischemic zone.

During the reentrant activity, one circuit (single rotor) of fairly large dimension was basically observed, i.e., the activity circles around an area of block the size of the ischemic area. For some CI's, double circuits were observed but not completed (or sustained), and the reentrant activity continued because of one single reentrant circuit. Patterns from our simulations were reported in the experimental work by Janse et al [6] in pig and dog hearts. For instance, Figure 8 in ref [6] shows single circuit and the eight shape reentrant pattern also obtained in our simulations (see Fig. 4). However, differently to the experimental studies by Janse et al., none of the cases studied lead to ventricular fibrillation. In all our simulations the reentrant activity was always restricted to the ischemic zone with clearly defined reentry circuits.

We have only monitored the reentrant activity up to five seconds during which we have observed perpetuating activity for a restricted vulnerable window. In some cases, i.e., $\mathrm{CI}=265 \mathrm{~ms}$ and $\mathrm{CI}=300$ $\mathrm{ms}$, the reentrant activity was spontaneously terminated after 3 seconds of activity, or before five seconds. This type of behavior was also reported in [6] where tachycardia terminated within $30 \mathrm{sec}$ after initiation. Additional studies on larger ischemic zones are required in order to determine if the size of the ischemic area may favor the onset of ventricular fibrillation in the ischemic heart. In addition, the location of the ectopic activity maybe important for both, the size of the vulnerable window and the reentrant pattern.

In conclusion, the model predicts the generation of reentry within the ischemic zone due to the heterogeneity in the refractory period between the ischemic affected area and the normal myocardium. The observed patterns obtained with the simulations are in good agreement with experimental studies conducted in pig and dog hearts subjected to acute regional ischemia.

\section{References}

1. Rubart M, Zipes DP. J Clin Invest 2005; 115(9):2305-2315.

2. Katz A. Physiology of the Heart. Lippincott Williams and Wilkins 2001.

3. Carmeliet E. Physiol Rev 1999; 79: 917-1017.

4. Janse MJ, Kleber AG. Circ Res 1981; 49(5): 1069-1081.

5. Coronel R. Cardiovasc. Res. 1994; 28(6):770-777.

6. Janse MJ, et al. Circ Res 1980; 47(2): 151-165.

7. Wilensky RL, et al. Circulation 1986; 74: 1137-1146.

8. Geselowitz DB, Miller III, WT. Ann Biomed Eng 1983; 11:315-334.

9. ten Tusscher KHWJ, Panfilov AV. Am J Physiol Heart Circ Physiol 2006; 291: H1088-H1 100.

10. Ferrero JM, et al. Circ. Res. 1996; 79(2):208-221.

11. B. Calvo, E. Peña (eds.), Patient-Specific Computational Modeling, Lecture Notes in Computational Vision and Biomechanics 5 . Dordrecht: Springer 2012: 81-104.

12. Coronel R, et al. Circulation 1988; 77(5):1125-1138.

13. Ferrero JM, et al. Int J Bif and Chaos 2003; 13: 3703-3715.

14. Yatani A, et al. J. Membr. Biol. 1984; 78(2):163-168.

15. Durrer D, et al. Circulation 1970; 41: 899-912.

16. Mena Tobar A, et al. Comp Phys Comm 2015; 196: 280-289. 